\title{
Understanding the needs of caregivers of persons with dementia: a scoping review
}

\author{
Francine N. F. R. Queluz, ${ }^{1,2}$ (D) Emily Kervin, ${ }^{1}$ Lori Wozney, ${ }^{3}$ Pamela Fancey, ${ }^{1}$ \\ Patrick J. McGrath, ${ }^{3,4}$ and Janice Keefe ${ }^{1}$ \\ ${ }^{1}$ Nova Scotia Centre on Aging, Mount Saint Vincent University, Halifax, NS, Canada \\ ${ }^{2}$ São Francisco University, Campinas, SP, Brazil \\ ${ }^{3}$ IWK Health Centre, Halifax, NS, Canada \\ ${ }^{4}$ Department of Psychiatry, Dalhousie University, Halifax, NS, Canada
}

ABSTRACT

Background: The number of people living with dementia (PWD) is increasing worldwide, corresponding with an increasing number of caregivers for PWD. This study aims to identify and describe the literature surrounding the needs of caregivers of PWD and the solutions identified to meet these needs.

Method: A literature search was performed in: PsycInfo, Medline, CINAHL, SCIELO and LILACS, January 2007-January 2018. Two independent reviewers evaluated 1,661 abstracts, and full-text screening was subsequently performed for 55 articles. The scoping review consisted of 31 studies, which were evaluated according to sociodemographic characteristics, methodological approach, and caregiver's experiences, realities, and needs. To help extract and organize reported caregiver needs, we used the C.A.R.E. Tool as a guiding framework.

Results: Thirty-one studies were identified. The most common needs were related to personal health (58\% emotional health; $32 \%$ physical health) and receiving help from others $(55 \%)$. Solutions from the articles reviewed primarily concerned information gaps $(55 \%)$ and the education/learning needs of caregivers (52\%).

Conclusion: This review identified the needs of caregivers of PWD. Caregivers' personal health emerged as a key area of need, while provision of information was identified as a key area of support. Future studies should explore the changes that occur in needs over the caregiving trajectory and consider comparing caregivers' needs across different countries.

Key words: caregivers, dementia, elderly, needs, demands, old person, care provider, Alzheimer's disease

\section{Introduction}

The prevalence of dementia is increasing as the global population ages. The World Health Organization (WHO) (2017) estimated that 47 million people currently live with dementia around the world and that this number will triple by the year 2050. Currently, most individuals living with dementia are cared for by families and friends (WHO, 2017), who are essential to the care recipient's quality of life (Farina et al., 2017).

Family and friend caregivers incur physical, psychological, social, and financial costs as part of their

Correspondence should be addressed to: Francine N. F. R. Queluz, Rua Lucio Pereira Peixoto, 95 Apto 57, Jardim Chapadão, CEP:13070-028- CampinasSP, Brazil. Phone: +55 1932910601; Fax: +55 1932910601. Email: francine. queluz@gmail.com. Received 10 Aug 2018; revision requested 30 Oct 2018; revised version received 31 Jan 2019; accepted 01 Feb 2019. First published online 10 April 2019. role and often experience stigmatization due in part to the lack of understanding surrounding dementia in the majority of countries (WHO, 2018). Caring for a loved one with dementia can be burdensome, and many caregivers suffer from reduced quality of life (Takai et al., 2011; Tomomitsu et al., 2014), limiting social engagement and support (Waligora, Bahouth \& Han, 2018), stress-related cognitive dysfunction (Oken et al., 2011), and depression and anxiety (Sörensen and Conwell, 2011; Laks et al., 2016). Evidence suggests that caring for a PWD is more burdensome than caring for persons living with other illnesses (Alzheimer's Association, 2012; Pinto and Barham, 2014). Research suggests that the prevalence of anxiety disorders is higher for caregivers of PWD than for other caregivers (Cooper et al., 2007) and that the likelihood of self-reported depression is higher for caregivers of persons with dementia than non-caregivers (Posner et al., 2015; 
Tomomitsu et al., 2014). When comparing caregivers of PWD to other caregivers, caregivers of PWD were found to have significantly worse subjective well-being and physical health (Brodaty and Donkin, 2009; Pinto and Barham, 2014). Taken together, these studies point to the importance of understanding the unique psychosocial needs of caregivers of PWD.

The needs of caregivers have been researched in the United Kingdom, United States, and Canada, and these studies have all called for agencies to assess caregivers' needs and provide them with targeted support and services. Usually, caregiver's needs relate to the physical, psychological, and social demands of providing assistance to the person with dementia. The C.A.R.E. (Caregivers' Aspirations, Realities and Expectations) Tool is a psychosocial assessment tool to identify and understand aspects of the caregiver's situation, such as their problems, strengths, and needs (Guberman et al., 2018, Keefe et al., 2008), and is used with multiple populations including caregivers of PWD.

Given decision-makers' growing interest in supporting PWD to age in place, a synthesis of existing evidence on PWDs' key source of support, family and friend caregivers, is essential. In the last decade, a handful of reviews surrounding the health and wellbeing of caregivers of PWD have been published. These reviews vary in objective, having examined the roles of caregiver self-efficacy (Crellin et al., 2014), meaning and motivation (Quinn et al., 2010), as well as psychological interventions that support caregivers of PWD (Elvish et al., 2013) and their quality of life (Farina et al., 2017). To date, reviews have typically examined caregiver needs at a particular time point; for example, during the care recipient's transition from home to long-term care (Afram et al., 2015). Others have introduced inclusion criteria that limit the review to certain research designs (e.g., McCabe et al., 2016), or are narrow in focus, such as Bangerter et al.'s (2017) a recent review of measurement strategies to assess the needs of caregivers of PWD.

While these reviews have contributed to new understandings, their restricted aims and inclusion criteria have led to a patchwork understanding of caregiver needs. To our knowledge, a broad overview of the literature including qualitative and quantitative studies has not been undertaken. In order to address this gap, a scoping review of primary research assessing the needs of caregivers of PWD was conducted. A more comprehensive understanding of caregivers needs and what recommendations are being made to address these needs are essential to supporting the development and enhancement of appropriate interventions to facilitate PWD to age in place.
Objective: To understand the needs of caregivers of persons with dementia and the recommendations being made to meet these needs. Questions guiding the review were: What do we know about the needs of caregivers of PWD? How are caregiver needs measured and described? What types of recommendations are being made about how to address caregivers' needs?

\section{Methods}

\section{Search strategy}

A literature search was performed in the following databases: PsycInfo, Medline, CINAHL, SCIELO, and LILACS to identify articles published between January 2007 and January 2018. The search was limited to peer-reviewed articles within the last 10 years to ensure that the current needs of caregivers of PWD were captured. Search terms were selected through consultation with a research librarian from standardized words compatible with Descriptors in Health Science (a trilingual thesaurus providing common terminology). Related review papers were also consulted. All searches were performed using the terms in English, Spanish, and Portuguese. Examples of key search terms included: "caregivers," "care providers," "elder care," "elderly," "old person," "care recipient," "dementia," "caregiver support," "needs," and "demands". For a complete list of search terms, see Table S1 published as supplementary material online attached to the electronic version of this paper at http://journals.cambridge.org/ipg.

\section{Study selection}

Studies were included in the review if they met the following criteria: (a) published in Portuguese, English, or Spanish; (b) published since 2007; (c) peer-reviewed primary research; (d) evaluated the needs of family caregivers; and (e) involved caregivers of PWD. Studies were excluded from this review if they: (a) did not address caregiver needs; (b) were not related to the caregiver's health; (c) addressed caregiving unrelated to people living with dementia; (d) focused only on the needs of the PWD; (e) were books, reviews, or meta-analyses.

The process used for collection, evaluation, and selection of the articles is represented in Figure 1, following the PRISMA recommendations for systematic reviews (Moher et al., 2009). Articles retrieved from each database were imported into Mendeley, and any duplicates were removed. Based on the inclusion and exclusion criteria, the abstract and title of the studies were screened by two reviewers independently (FQ, EK). The reviewers 


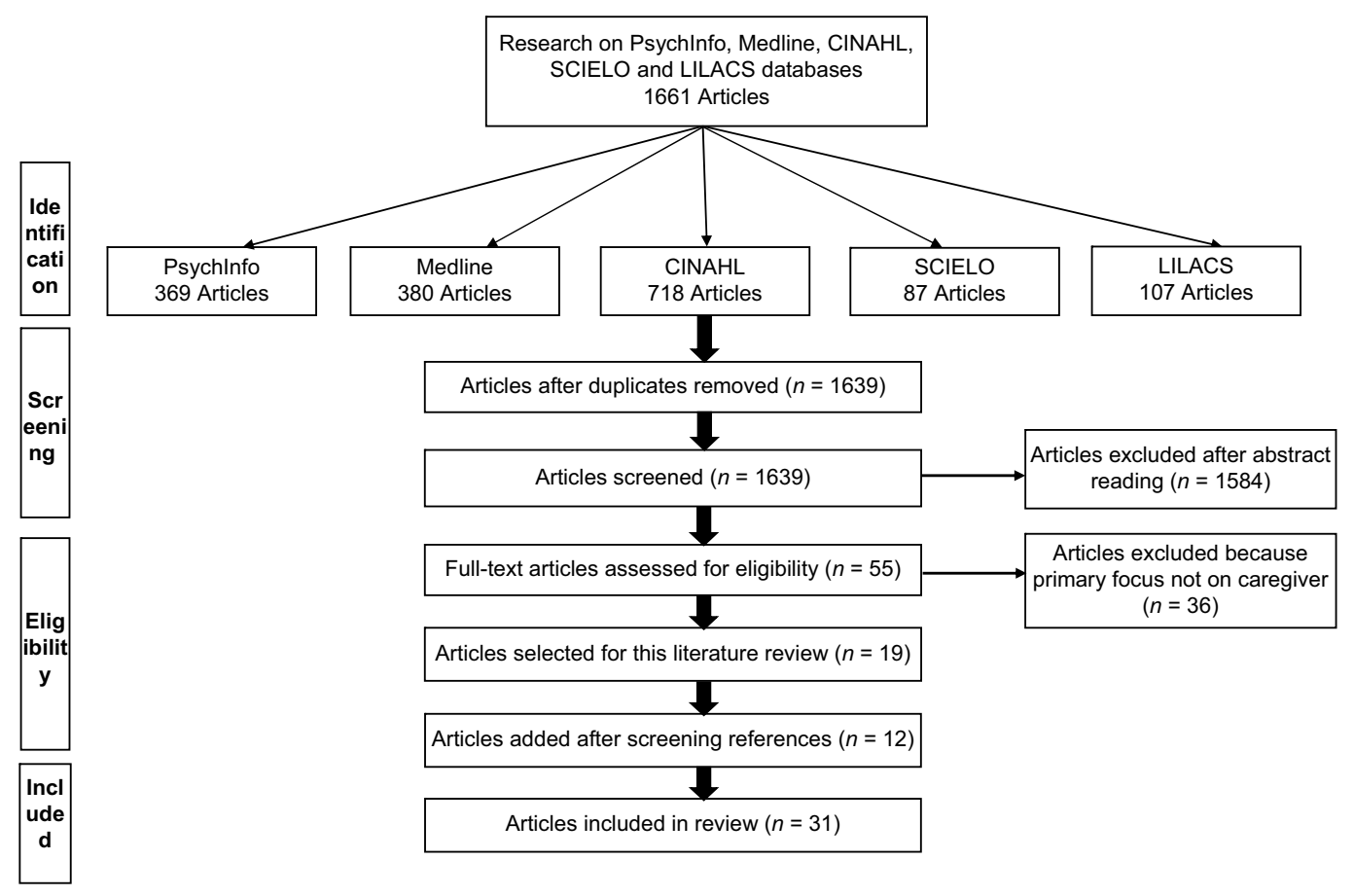

Figure 1. PRISMA flow diagram (Moher et al., 2009) illustrating an overview of the search strategy.

obtained $98 \%$ agreement; disagreements during title and abstract screening were resolved through further discussion between the same two reviewers. The full texts of identified studies $(n=55)$ were then reviewed independently by the same two reviewers. The reviewers reached $100 \%$ agreement in independent evaluation during full-text screening and 36 articles were excluded. The reference lists of the remaining 19 articles and of the 5 systematic reviews that were found in the first screening were consulted to identify other possible studies. This search yielded 12 additional articles published in journals not indexed in the databases included in the original search. A total of 31 studies were selected for this review.

\section{Data extraction and synthesis}

The coding was completed collaboratively by $\mathrm{FQ}$ and EK, and any disagreements/questions requiring further discussion were resolved in team meetings. A combination of deductive and inductive coding strategies was used according to Bardin's (1977) categorization; thus, approach decisions were grounded in existing categorization schemes to sort and organize information. In this review, the data were organized considering the relevance of the content and if the content obeyed the inclusion and exclusion criteria. The focus was on articles that studied the caregiver's needs including the physical, psychological, and social demands of providing assistance to the person with dementia.
However, attention was also given to identifying novel or nuanced data points that appeared salient to answering the review questions. Coding strategies were refined several times throughout the process. The review team held weekly meetings to refine coding procedures and promote methodological rigor through discussion.

A data extraction spreadsheet was designed to capture the pertinent characteristics of the reviewed studies. Major data extraction categories included: (1) sociodemographic characteristics (e.g. gender, age, living arrangement, country of the study, and caregiver's relationship to PWD); (2) methodological approach (e.g. research design and sample size); and (3) the eight domains of the C.A.R.E. Tool as representing caregivers' experiences, realities, and needs (Keefe et al., 2008). The tool consists of eight evidence-based domains providing a comprehensive view of caregivers' situational needs and areas where support could be targeted: caregiving tasks, relationship with formal service providers, housing, juggling responsibilities, financial costs, personal health, relationships, and planning. Finally, authors' recommendations for addressing the needs of caregivers as identified in each of the 31 studies were extracted.

After extraction was completed, FQ and EK conducted a descriptive analysis (frequencies, means) where applicable and organized the authors' recommendations for addressing caregivers' needs into broad themes. Thematic categories were reviewed with the team and refined for clarity. 
Consistent with the exploratory nature of scoping reviews and typical methodological approaches applied in other published scoping reviews, we did not conduct a quality assessment of studies (Arksey and O’Malley, 2005).

\section{Results}

\section{Socio-demographic and methodological characteristics}

Most studies were conducted in Europe $(n=14)$ and North America $(n=9)$, with the remaining taking place in Thailand $(n=2)$, Brazil, Japan, Australia, and China. One study was conducted across Europe (Kerpershoek et al., 2017). Although the selection criteria allowed for articles written in Portuguese, English, and Spanish, all included articles had a version available in English. A total of 7,533 caregivers was captured in the included studies. Study sample sizes ranged from 9 (Jong and Boersma, 2009) to 1,494 (Zwaanswijk et al., 2013), with $42 \%$ of studies involving less than 100 participants and $19 \%$ of studies involving 20 participants or less. In all but one study (Millenaar et al., 2018), the proportion of female caregivers was higher than the proportion of male caregivers. Caregivers were most often the spouse/partner of the PWD, but in $26 \%$ of the studies, the largest caregiver group was adult children. Caregivers were typically more than 50 years of age. In $39 \%$ of the studies, the living arrangement of caregiver and PWD was reported, and only three (Galvin et al., 2011; Jong and Boersma, 2009; Landmark et al., 2013) indicated that most caregivers were not co-residing. Most studies did not specify the care recipient's type of dementia (65\%). In the 10 studies that did report this information, the majority of care recipients had Alzheimer's disease.

In $35 \%$ of the studies, the primary data on caregiver needs was collected with the aid of questionnaires. General descriptions of interviews and focus groups suggested that researchers typically asked open-ended questions to caregivers about their primary needs as caregivers. Four studies reported using a published instrument to evaluate caregiver needs; studies by Nicolaou et al. (2010) and Kerpershoek et al. (2017) used the Camberwell Assessment of Need for the Elderly (CANE) (Orrell and Hancock, 2004), and studies by Hughes et al. (2014) and Black et al. (2013) used the Johns Hopkins Dementia Care Needs Assessment (JHDCNA) (Black et al., 2008). See Table 1 for complete information.

\section{Caregivers' needs}

Needs reported in primary studies were dispersed across many domains, with only three domains consistently identified across more than $50 \%$ of studies: caregivers' emotional health (58\%), formal or informal help received from others (55\%), and information about dementia and dementia care $(52 \%)$. The emotional health domain encompassed caregivers' need for assistance with managing emotional strain due to concern for the PWD (Cascioli et al., 2008); the need to be recognized as a caregiver by others (Wawrznicsky et al., 2017); and to be supported when discussing one's emotions with friends, family, and professional counsellors (Vaingankar et al., 2013). Studies that identified a need for formal or informal help from others found that the absence of family support in caring for the PWD could result in inadequate time for the caregiver to get sufficient sleep or to engage in leisure activities (e.g., Gratão et al. 2010; Landmark et al., 2013). Caregivers participating in focus groups in Landmark et al. (2013) expressed feelings of disappointment, frustration, grief, and anger regarding family members who refused to support them, and authors in three studies (Kerpershoek et al., 2017; Landmark et al., 2013; Lilly et al., 2012) suggested that this lack of support can directly affect the caregiver's physical and mental health. For caregivers whose needs involved information about dementia, information specifically about the progression of dementia and what behavior changes to expect throughout the disease trajectory seemed to be especially important (Diehl-Schmid et al., 2013).

Several studies found needs surrounding physical health $(32 \%)$, professional support and formal services $(29 \%)$, and relationships with formal service providers $(29 \%)$. Needs surrounding physical health included having the time to take care of oneself and one's own health concerns (Wawrznicsky et al., 2017) and managing the physical burden of providing care and supervision for the PWD (Galvin et al., 2011). In relation to formal support, caregivers in many studies suggested that they would like to receive more information about services available nearby and the costs associated with these services (Raivio et al., 2007; Vaingankar et al., 2013). In some studies, caregivers expressed a desire to find information all in the same place (e.g., Cascioli et al., 2008; Diehl-Schmid et al., 2013). The literature pertaining to caregivers' relationships with formal service providers suggested a need for caregivers' concerns to be addressed separately from those of the PWD and to have opportunities to address sensitive issues without the PWD present (Cascioli et al., 2008). There was also a need for a good "fit" with the service provider, as almost half of respondents in one study believed that their provider(s) did not understand their needs (Gibson et al., 2014). The C.A.R.E Tool domains that were found the least frequently in primary studies included 
Table 1. Socio-demographic and methodological characteristics of the articles by year of publication

\begin{tabular}{|c|c|c|c|c|c|c|c|c|c|}
\hline $\begin{array}{l}\text { STUDY } \\
\text { (PUB YEAR) }\end{array}$ & COUNTRY & $\begin{array}{l}\text { DATA } \\
\text { COLLECTION }\end{array}$ & $\begin{array}{l}\text { METHOD OF } \\
\text { ADMINISTRATION }\end{array}$ & $\begin{array}{l}\text { SAMPLE } \\
\text { SIZE (CG) }\end{array}$ & $\begin{array}{l}\text { CAREGIVER AGE } \\
\text { (MEAN IN YRS) }\end{array}$ & GENDER & $\begin{array}{l}\text { RELATIONSHIP } \\
\text { WITH PERSON } \\
\text { WITH DEMENTIA }\end{array}$ & $\begin{array}{l}\text { DEMENTIA } \\
\text { TYPE }\end{array}$ & $\begin{array}{l}\text { CAREGIVER'S } \\
\text { LIVING } \\
\text { ARRANGEMENT } \\
(\%)\end{array}$ \\
\hline $\begin{array}{l}\text { Lai and } \\
\text { Chung } \\
\text { (2007) }\end{array}$ & China & Q & $\begin{array}{l}\text { In person/Mail } \\
\text { Self-administered }\end{array}$ & 144 & $\begin{array}{l}\text { Mean not } \\
\text { specified }^{*}\end{array}$ & $\begin{array}{l}\text { F } 63 \% \\
\text { M 33\% } \\
4 \% \text { did not } \\
\text { report }\end{array}$ & $\begin{array}{l}\text { Spouse }(13 \%), \\
\text { adult children } \\
(66 \%) \text {, other } \\
\text { relatives }(19 \%) \text {, } \\
\text { and parent } \\
(1 \%) .\end{array}$ & Not reported. & Not reported. \\
\hline $\begin{array}{l}\text { Raivio et al. } \\
\quad(2007)\end{array}$ & Finland & Q & $\begin{array}{l}\text { Mail } \\
\text { Self-administered }\end{array}$ & 1214 & 78 & $\begin{array}{l}\text { F } 63 \% \\
\text { M 37\% }\end{array}$ & Spouse $(100 \%)$ & Not reported. & Not reported. \\
\hline $\begin{array}{l}\text { Cascioli et al. } \\
\text { (2008) }\end{array}$ & Wales & SSI & $\begin{array}{l}\text { In person } \\
\text { Interviewer- } \\
\text { administered }\end{array}$ & 45 & 61 & $\begin{array}{l}\text { F } 69 \% \\
\text { M } 31 \%\end{array}$ & $\begin{array}{l}\text { Spouse }(53 \%) \text { and } \\
\text { adult children } \\
(47 \%) .\end{array}$ & $\begin{array}{l}\text { AD }(40 \%), \text { other } \\
\text { types }(20 \%) \\
\text { and without } \\
\text { definitive } \\
\text { diagnosis } \\
(40 \%) .\end{array}$ & Not reported. \\
\hline $\begin{array}{l}\text { Lawrence et al. } \\
\quad \text { (2008) }\end{array}$ & $\begin{array}{l}\text { United } \\
\text { Kingdom }\end{array}$ & IDI & $\begin{array}{l}\text { In person } \\
\text { Interviewer- } \\
\text { administered }\end{array}$ & 32 & $\begin{array}{l}\text { Mean not } \\
\text { specified }\end{array}$ & $\begin{array}{l}\text { F } 78 \% \\
\text { M } 22 \%\end{array}$ & $\begin{array}{l}\text { Spouses or adult } \\
\text { children (\% not } \\
\text { specified). }\end{array}$ & Not reported. & Not reported. \\
\hline $\begin{array}{l}\text { Jong and } \\
\text { Boersma } \\
(2009)\end{array}$ & Netherlands & SSI & $\begin{array}{l}7 \text { in person } \\
2 \text { by phone } \\
\text { Interviewer- } \\
\text { administered }\end{array}$ & 9 & 76 & $\begin{array}{l}\text { F } 55 \% \\
\text { M } 44 \%\end{array}$ & $\begin{array}{l}\text { Spouse }(33 \%) \text { and } \\
\text { adult children } \\
(67 \%) .\end{array}$ & Not reported. & $\begin{array}{l}\text { Co-residing } \\
(33 \%)\end{array}$ \\
\hline $\begin{array}{l}\text { Nichols et al. } \\
\text { (2009) }\end{array}$ & $\begin{array}{l}\text { United } \\
\text { States }\end{array}$ & $\mathrm{Q}^{*}$ & In person & 165 & 65 & $\begin{array}{l}\text { F 55\% } \\
\text { M 45\% }\end{array}$ & $\begin{array}{l}\text { Spouse }(50 \%), \\
\text { adult children } \\
(35 \%) \text { and } \\
\text { other }(15 \%) .\end{array}$ & Not reported. & Not reported. \\
\hline $\begin{array}{l}\text { Galvin et al. } \\
\text { (2011) }\end{array}$ & $\begin{array}{l}\text { United } \\
\text { States }\end{array}$ & $\mathrm{S}$ & Online & 962 & 56 & $\begin{array}{l}\text { F } 88 \% \\
\text { M } 12 \%\end{array}$ & $\begin{array}{l}\text { Spouse }(41 \%) \text {, } \\
\text { adult child } \\
(52 \%) \text { and } \\
\text { other }(7 \%)\end{array}$ & LB $(100 \%)$ & $\begin{array}{l}\text { Co-residing } \\
(46 \%) .\end{array}$ \\
\hline $\begin{array}{l}\text { Gratão et al. } \\
\quad(2010)\end{array}$ & Brazil & IT & In person & 90 & 52 & $\begin{array}{l}\text { F } 80 \% \\
\text { M } 20 \%\end{array}$ & $\begin{array}{l}\text { Not specified. } \\
\text { Family member } \\
(90 \%) \text {. }\end{array}$ & $\begin{array}{l}\text { AD }(53 \%), \mathrm{VD} \\
(10 \%), \\
\mathrm{AD}+\mathrm{VD} \\
(11 \%), \text { FTD } \\
(2 \%), \mathrm{LB}(2 \%) \\
\text { Unknown } \\
(8 \%), \text { Other } \\
(14 \%) .\end{array}$ & Not reported. \\
\hline
\end{tabular}


Table 1. Continued

\begin{tabular}{|c|c|c|c|c|c|c|c|c|c|}
\hline $\begin{array}{l}\text { STUDY } \\
\text { (PUB YEAR) }\end{array}$ & COUNTRY & $\begin{array}{l}\text { DATA } \\
\text { COLLECTION }\end{array}$ & $\begin{array}{l}\text { METHOD OF } \\
\text { ADMINISTRATION }\end{array}$ & $\begin{array}{l}\text { SAMPLE } \\
\text { SIZE (CG) }\end{array}$ & $\begin{array}{l}\text { CAREGIVER AGE } \\
\text { (MEAN IN YRS) }\end{array}$ & GENDER & $\begin{array}{l}\text { RELATIONSHIP } \\
\text { WITH PERSON } \\
\text { WITH DEMENTIA }\end{array}$ & $\begin{array}{l}\text { DEMENTIA } \\
\text { TYPE }\end{array}$ & $\begin{array}{l}\text { CAREGIVER'S } \\
\text { LIVING } \\
\text { ARRANGEMENT } \\
(\%)\end{array}$ \\
\hline $\begin{array}{l}\text { Czarnuch and } \\
\text { Mihailidis } \\
\text { (2011) }\end{array}$ & Canada & $\mathrm{Q}$ & Online & 106 & $\begin{array}{l}\text { Mean not } \\
\text { specified }\end{array}$ & $\begin{array}{l}\text { Not } \\
\quad \text { reported. }\end{array}$ & $\begin{array}{l}\text { Partner }(28 \%), \\
\text { adult children } \\
(35 \%) \text {, family } \\
\text { member }(34 \%), \\
\text { friend }(2 \%) \text { and } \\
\text { other }(1 \%) \text {. }\end{array}$ & Not reported. & $\begin{array}{c}\text { Co-residing } \\
(59 \%)\end{array}$ \\
\hline $\begin{array}{l}\text { Hirakawa } \\
\text { et al. (2011) }\end{array}$ & Japan & Q & Self-administered & 475 & 65 & $\begin{array}{l}\text { F } 77 \% \\
\text { M } 23 \%\end{array}$ & $\begin{array}{l}\text { Spouse }(43 \%) \text {, } \\
\text { adult children } \\
(49 \%) \text { and others } \\
(6 \%) .\end{array}$ & Not reported. & $\begin{array}{c}\text { Co-residing } \\
(89 \%)\end{array}$ \\
\hline $\begin{array}{l}\text { Lilly et al. } \\
\quad(2012)\end{array}$ & Canada & FG & $\begin{array}{l}\text { In person } \\
\text { Interviewer- } \\
\text { administered }\end{array}$ & 15 & 66 & $\begin{array}{l}\text { F } 87 \% \\
\text { M } 13 \%\end{array}$ & $\begin{array}{l}\text { Spouse }(53 \%), \\
\text { adult children } \\
(47 \%) \text { and/or } \\
\text { other }(10 \%) .\end{array}$ & Not reported. & Not reported. \\
\hline $\begin{array}{l}\text { McHugh } \\
\quad \text { et al. }(2012)\end{array}$ & Ireland & SSI & $\begin{array}{l}\text { In person } \\
\text { Interviewer- } \\
\text { administered }\end{array}$ & 13 & $\begin{array}{l}\text { Mean not } \\
\text { specified }\end{array}$ & $\begin{array}{l}\text { F } 61 \% \\
\text { M } 38 \%\end{array}$ & Spouse $(100 \%)$ & Not reported. & Not reported. \\
\hline $\begin{array}{l}\text { Samia } \\
\quad \text { et al. (2012) }\end{array}$ & $\begin{array}{l}\text { United } \\
\text { States }\end{array}$ & $\mathrm{S}+\mathrm{FG}$ & $\begin{array}{l}\text { In person }(\mathrm{FG}) \\
\text { Interviewer- } \\
\text { administered } \\
\text { Self-administered }(\mathrm{S})\end{array}$ & $\begin{array}{l}168(\mathrm{~S}) \\
26(\mathrm{FG})\end{array}$ & 67 & $\begin{array}{l}\text { F } 84 \% \\
\text { M 16\% }\end{array}$ & $\begin{array}{l}\text { Spouse }(68 \%), \\
\text { adult children } \\
(31 \%) \text { and } \\
\text { other }(11 \%) .\end{array}$ & Not reported. & $\begin{array}{l}\text { Co-residing } \\
(>50 \% * * *)\end{array}$ \\
\hline $\begin{array}{l}\text { Diehl-Schmid } \\
\text { et al. }(2013)\end{array}$ & Germany & $\mathrm{Q}+\mathrm{IT}$ & $\begin{array}{l}\text { Mail (Q) } \\
\text { Self-administered } \\
\text { Telephone (IT) }\end{array}$ & 94 & 59 & $\begin{array}{l}\text { F } 72 \% \\
\text { M } 28 \%\end{array}$ & $\begin{array}{l}\text { Spouse }(80 \%) \text {, } \\
\text { child in law } \\
(12 \%) \text { and } \\
\text { other }(8 \%) .\end{array}$ & $\begin{array}{c}\text { FTD }(87 \%) \text { and } \\
\text { other }(13 \%) .\end{array}$ & $\begin{array}{c}\text { Co-residing } \\
\quad(72 \%)\end{array}$ \\
\hline
\end{tabular}




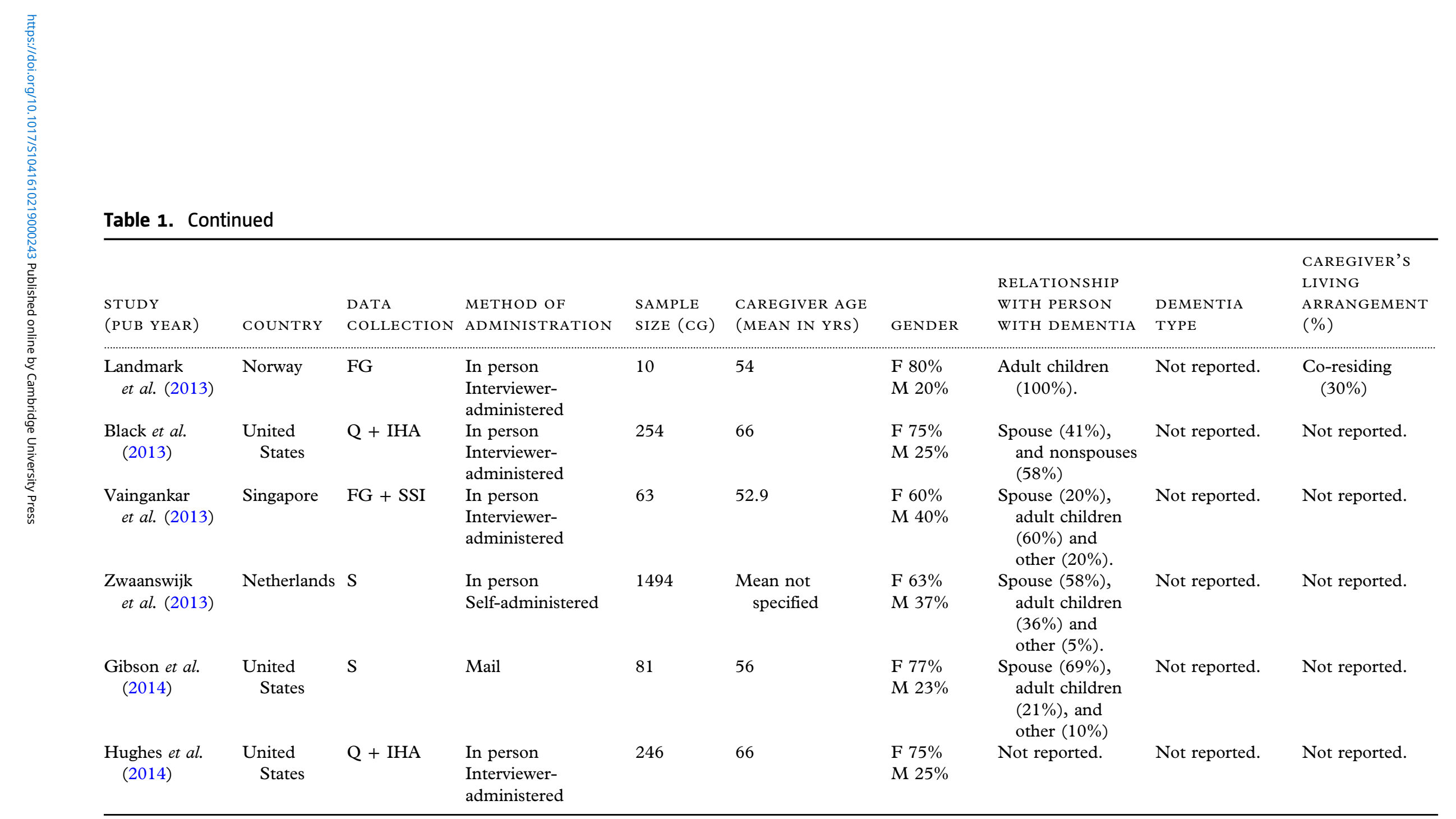


Table 1. Continued

\begin{tabular}{|c|c|c|c|c|c|c|c|c|c|}
\hline $\begin{array}{l}\text { STUDY } \\
\text { (PUB YEAR) }\end{array}$ & COUNTRY & $\begin{array}{l}\text { DATA } \\
\text { COLLECTION }\end{array}$ & $\begin{array}{l}\text { METHOD OF } \\
\text { ADMINISTRATION }\end{array}$ & $\begin{array}{l}\text { SAMPLE } \\
\text { SIZE (CG) }\end{array}$ & $\begin{array}{l}\text { CAREGIVER AGE } \\
\text { (MEAN IN YRS) }\end{array}$ & GENDER & $\begin{array}{l}\text { RELATIONSHIP } \\
\text { WITH PERSON } \\
\text { WITH DEMENTIA }\end{array}$ & $\begin{array}{l}\text { DEMENTIA } \\
\text { TYPE }\end{array}$ & $\begin{array}{l}\text { CAREGIVER'S } \\
\text { LIVING } \\
\text { ARRANGEMENT } \\
(\%)\end{array}$ \\
\hline $\begin{array}{l}\text { Boots et al. } \\
\quad(2015)\end{array}$ & Netherlands & FG & $\begin{array}{l}\text { In person } \\
\text { Interviewer- } \\
\text { administered }\end{array}$ & 28 & 64 & $\begin{array}{l}\text { F } 75 \% \\
\text { M 25\% }\end{array}$ & $\begin{array}{l}\text { Spouse }(78 \%), \\
\text { adult children } \\
(7 \%), \text { child in } \\
\text { law }(7 \%), \\
\text { sibling }(4 \%) \\
\text { and friend } \\
(4 \%) .\end{array}$ & $\begin{array}{l}\text { MCI }(32 \%), \text { AD } \\
\quad(44 \%), \text { other } \\
\quad(24 \%) .\end{array}$ & $\begin{array}{l}\text { Co -residing } \\
\quad(75 \%)\end{array}$ \\
\hline $\begin{array}{l}\text { Griffiths and } \\
\text { Bunrayong } \\
(2016)\end{array}$ & Thailand & SSI & $\begin{array}{l}\text { In person } \\
\text { Interviewer- } \\
\text { administered }\end{array}$ & 30 & $\begin{array}{l}\text { Mean not } \\
\text { specified }\end{array}$ & $\begin{array}{l}\text { F } 80 \% \\
\text { M 20\% }\end{array}$ & $\begin{array}{l}\text { Spouse }(40 \%), \\
\text { adult children } \\
(46 \%), \text { and } \\
\text { other }(14 \%) \text {. }\end{array}$ & Not reported. & Not reported. \\
\hline $\begin{array}{l}\text { Peterson et al. } \\
\quad(2016)\end{array}$ & $\begin{array}{l}\text { United } \\
\text { States }\end{array}$ & IDI & Unclear & 27 & 59 & $\begin{array}{l}\text { F } 70 \% \\
\text { M } 30 \%\end{array}$ & $\begin{array}{l}\text { Spouse }(30 \%) \text { and } \\
\text { adult children } \\
(56 \%) \text { and } \\
\text { others }(14 \%) .\end{array}$ & $\begin{array}{l}\text { NFD }(60 \%), \\
\text { DUC }(21 \%), \\
\text { AD }(11 \%), \text { LB } \\
(4 \%), \text { PD }(4 \%), \\
\text { other }\end{array}$ & Not reported. \\
\hline $\begin{array}{l}\text { Kerpershoek } \\
\text { et al. }(2017)\end{array}$ & $\begin{array}{c}\text { Netherlands, } \\
\text { Germany, } \\
\text { United } \\
\text { Kingdom, } \\
\text { Ireland, } \\
\text { Sweden, } \\
\text { Norway, } \\
\text { Portugal } \\
\text { and Italy }\end{array}$ & & $\begin{array}{l}\text { In person } \\
\text { Interviewer- } \\
\text { administered }\end{array}$ & 451 & 66 & $\begin{array}{l}\text { F } 67 \% \\
\text { M 33\% }\end{array}$ & $\begin{array}{l}\text { Spouse }(60 \%) \\
\text { adult children } \\
(30 \%) \text { and } \\
\text { other }(10 \%) \text {. }\end{array}$ & $\begin{array}{l}\operatorname{AD}(49 \%), \\
\operatorname{VD}(12 \%), \mathrm{MD} \\
(12 \%), \\
\text { LB }(1 \%) \text { and } \\
\text { other }(26 \%) .\end{array}$ & $\begin{array}{l}\text { Co-residing } \\
\quad(72 \%)\end{array}$ \\
\hline
\end{tabular}




\begin{tabular}{|c|c|c|c|c|c|c|c|c|c|}
\hline $\begin{array}{l}\text { STUDY } \\
\text { (PUB YEAR) }\end{array}$ & COUNTRY & $\begin{array}{l}\text { DATA } \\
\text { COLLECTION }\end{array}$ & $\begin{array}{l}\text { METHOD OF } \\
\text { ADMINISTRATION }\end{array}$ & $\begin{array}{l}\text { SAMPLE } \\
\text { SIZE (CG) }\end{array}$ & $\begin{array}{l}\text { CAREGIVER AGE } \\
\text { (MEAN IN YRS) }\end{array}$ & GENDER & $\begin{array}{l}\text { RELATIONSHIP } \\
\text { WITH PERSON } \\
\text { WITH DEMENTIA }\end{array}$ & $\begin{array}{l}\text { DEMENTIA } \\
\text { TYPE }\end{array}$ & $\begin{array}{l}\text { CAREGIVER'S } \\
\text { LIVING } \\
\text { ARRANGEMENT } \\
(\%)\end{array}$ \\
\hline $\begin{array}{l}\text { Wawrziczny } \\
\text { et al. (2017) }\end{array}$ & France & SSI & $\begin{array}{l}\text { In person } \\
\text { Interviewer- } \\
\text { administered }\end{array}$ & 78 & $\begin{array}{l}\text { 57(Group 1) } \\
77(\text { Group 2) }\end{array}$ & $\begin{array}{l}\text { F 58\% } \\
\text { M 42\% } \\
\text { (Group 1) } \\
\text { F 53\% } \\
\text { M 47\% } \\
\text { (Group 2) }\end{array}$ & Spouse $(100 \%)$ & $\begin{array}{l}\mathrm{AD}(50 \%), \mathrm{MD} \\
(45 \%), \mathrm{LB} \\
(5 \%) \text { (Group 1) } \\
\text { and AD (60\%), } \\
\text { FTD (23\%), } \\
\text { MD (12\%), and } \\
\text { LB (5\%) } \\
\text { (Group 2). }\end{array}$ & $\begin{array}{l}\text { Co-residing } \\
(100 \%) \\
\end{array}$ \\
\hline $\begin{array}{l}\text { Millenaar et al. } \\
\quad(2018)\end{array}$ & Netherlands & $\mathrm{IT}+\mathrm{Q}$ & $\begin{array}{l}\text { In person } \\
\text { Interviewer- } \\
\text { administered }\end{array}$ & 18 & 59 & $\begin{array}{l}\text { F } 50 \% \\
\text { M } 50 \%\end{array}$ & $\begin{array}{l}\text { Spouse }(78 \%), \\
\text { adult children } \\
(11 \%) \text { and } \\
\text { friends }(11 \%) .\end{array}$ & Not reported. & Not reported. \\
\hline
\end{tabular}

Key code: Type of dementia $-\mathrm{AD}=\mathrm{Alzheimer}$ disease; FTD = Frontotemporal; VD = Vascular dementia; $\mathrm{MD}=\mathrm{Mixed}$ dementia; $\mathrm{LB}=\mathrm{Lewy}$ body dementia; $\mathrm{PD}=\mathrm{Parkinson}$ 'S disease; $\mathrm{DUC}=$
Dementia of an unknown cause $\mathrm{NFD}=$ No formal diagnosis; Method $-\mathrm{SSI}=$ Semi-structured interview; $\mathrm{IDI}=\mathrm{In}$-depth interview; IT = Interview (nonspecific); FG = Focus group; $\mathrm{Q}=\mathrm{Ques}-$ tionnaire; SA = Secondary Analysis; S = Survey, IHA = In-home assessment

* Although authors may have provided age range or age category frequencies, they did not specify explicitly the mean age of caregivers.

$\star \star$ Secondary analysis of questionnaire data.

$\star \star \star$ Reported in the study as "most." 
difficulty in care coordination (3\%), housing (10\%), and household work (10\%). Planning for the future $(23 \%)$ was cited more often than planning for crises $(13 \%)$. None of the primary studies reported needs that were categorized as juggling responsibilities. See Table 2 for complete information.

\section{Suggested solutions to address needs}

Studies concerning caregivers' needs appear to use "needs" as an umbrella term for programs, services, and information identified by caregivers (Griffiths and Bunrayong, 2016; Wawrznicsky et al., 2017). Often there is no clear distinction between needs that are met and needs that are unmet, although a handful of studies we reviewed do focus specifically on caregivers' unmet needs (Black et al., 2013; Hughes et al., 2014; Millenaar et al., 2018; Stirling et al., 2010; Vangainkar et al., 2013). Based on this narrow segment of the literature, it is possible that some needs are met less often than others, such as the need for referrals to appropriate community resources (Black et al., 2013) and obtaining adequate support for one's emotional health (Vaingankar et al., 2013).

Primary authors proposed many possible solutions to address the needs of caregivers identified in their studies. Two overarching themes emerged: improved educational and informational resources and expansion of formal services. For example, the provision of psychoeducational activities tailored to the stage of dementia (52\%) and information about coping with behavioral problems and changes in PWD (55\%) were the most frequent informationfocused recommendations (See Table 3). Three studies $(10 \%)$ recommended that information resources, including self-care for caregivers, be delivered in one place for easier access. Authors put forward an array of potentially relevant information topics: dementia (Hirakawa et al., 2011; Lai and Chung, 2007), first aid (Hirakawa et al., 2011), public long-term care services (Hirakawa et al., 2011; Lilly et al., 2012), food and nutrition (Hirakawa et al., 2011; Nicolaou et al., 2010), chronic and/or life-altering conditions (Lilly et al., 2012), stress management (Hirakawa et al., 2011; Rosa et al., 2010), and when to take the care recipient to a hospital (Nichols et al., 2009; Vaingankar et al., 2013), among others.

In addition to informational supports, study authors also made recommendations related to the provision of formal support to family caregivers, generally $(52 \%)$, services specifically directed to caregivers' emotional health $(42 \%)$ and respite/daycare services for PWD (23\%) in order to give caregivers more personal time. One study by Nicolaou et al. (2010) further recommended that services be differentiated according to type of dementia.

\section{Discussion}

The objective of this scoping review was to map the most current literature surrounding the needs of caregivers of PWD, the characteristics of studies in which needs have been assessed, and the emerging solutions proposed to meet these needs.

\section{What are the defining characteristics of studies reporting on the needs of caregivers of PWD?}

The results found in this scoping review are consistent with recent findings (Bangerter et al., 2017; Mansfield et al., 2017) that many instruments used to identify the unmet needs of caregivers of people with dementia lack indices of psychometric properties. The two published instruments identified in our review are in the initial phase of validation, and the remaining studies provided limited insights into instrumentation for interviews and focus groups. This suggests caution when interpreting results, as measures may lack consistency or theoretical stability. This review is among the first to apply recommendations for bringing attention to the conceptualization and classification of caregivers' needs to build a stronger foundation for measurement (Bangerter et al., 2017). By applying the validated psycho-social assessment C.A.R.E Tool as an organizing framework for data extraction, we captured both frequently and infrequently reported needs. An important caveat to this approach, however, is that the frequency of studies reporting on C.A.R.E Tool domains does not infer that those needs are viewed as more important by caregivers; therefore, the current literature may not align with caregivers' priority needs. Furthermore, each person's unique caregiving context will prompt different needs that may vary across situations and throughout the caregiving trajectory, demonstrating the merit of person-centered methods of assessment and treatment. The findings of this review do point to important new lines of research that could map some of the variations among caregivers' needs, including the intensity of caregivers' needs-their perceived urgency, level of burden, or clustering of needs over time, as well as across different types of dementia. Some preliminary work by Whitlatch and Orsulic-Jeras (2018) that mapped resources for caregivers from early-stage diagnosis until end of life is a practical example of trying to capture the dynamically evolving needs of caregivers. 
Table 2. Frequency analysis of caregiver needs identified in primary studies according to C.A.R.E. Tool domains $(N=31)$

\begin{tabular}{|c|c|c|}
\hline NEEDS OR DEMANDS & \# OF STUDIES (\%) & REFERENCES \\
\hline \multicolumn{3}{|l|}{ 1. Caregiving Tasks } \\
\hline a. Physical/nursing care; & $5(16 \%)$ & $\begin{array}{l}\text { Cascioli et al., 2008; Raivio et al., 2007; Rosa et al., 2010; Vaingankar et al., } \\
\text { 2013; Wawrziczny et al., } 2017\end{array}$ \\
\hline b. Household work; & $3(10 \%)$ & Raivio et al., 2007; Wawrziczny et al., 2017; Zwaanswijk et al., 2013 \\
\hline c. Supervision/ support; & $6(19 \%)$ & $\begin{array}{l}\text { Galvin et al., 2011; Lai and Chung 2007; Landmark et al., 2013; } \\
\text { Muangpaisan et al., 2010; Peeters et al., 2010; Wawrziczny et al., } 2017\end{array}$ \\
\hline d. Coordination; & $1(3 \%)$ & Rosa et al., 2010 \\
\hline $\begin{array}{l}\text { e. Help received from } \\
\text { others (informal \& } \\
\text { formal) }\end{array}$ & $17(55 \%)$ & $\begin{array}{l}\text { Black et al., 2013; Lai and Chung, 2007; Czarnuch and Mihailidis, 2011; } \\
\text { Gibson et al., 2014; Griffiths and Bunyarong, 2016; Hughes et al., 2014; } \\
\text { Lawrence et al., 2008; Lilly et al., 2012; Millenaar et al., 2018; } \\
\text { Muangpaisan et al., 2010; Raivio et al., 2007; Samia et al., 2012; } \\
\text { Stirling et al., 2010; Vaingankar et al., 2013; Wawrziczny et al., 2017; } \\
\text { Zwaanswijk et al., } 2013\end{array}$ \\
\hline $\begin{array}{l}\text { 2. Relationship with Formal } \\
\text { Service Providers }\end{array}$ & $9(29 \%)$ & $\begin{array}{l}\text { Cascioli et al., 2008; Diehl-Schmid et al., 2013; Gibson et al., 2014; Lilly } \\
\text { et al., 2012; McHugh et al., 2012; Millenaar et al., 2018; Samia et al., } \\
\text { 2012; Vaingankar et al., 2013; Wawrziczny et al., } 2017\end{array}$ \\
\hline 3. Housing & $3(10 \%)$ & $\begin{array}{l}\text { Vaingankar et al., 2013; Griffiths and Bunyarong, 2016; Muangpaisan } \\
\text { et al., } 2010\end{array}$ \\
\hline 4. Juggling Responsibilities & $0(\%)$ & \\
\hline 5. Financial Costs & $6(19 \%)$ & $\begin{array}{l}\text { Cascioli et al., 2008; Diehl-Schmid et al., 2013; Gibson et al., 2014; } \\
\text { Griffiths and Bunyarong, 2016; Raivio et al., 2007; Vaingankar } \\
\text { et al., } 2013\end{array}$ \\
\hline \multicolumn{3}{|l|}{ 6. Personal Health } \\
\hline a. Physical health & $10(32 \%)$ & $\begin{array}{l}\text { Black et al., 2013; Cascioli et al., 2008; Galvin et al., 2011; Gratão et al., } \\
\text { 2010; Hirakawa et al., 2011; Hughes et al., 2014; Landmark et al., 2013; } \\
\text { Nichols et al., 2009; Samia et al., 2012; Wawrziczny et al., } 2017\end{array}$ \\
\hline b. Emotional health & $18(58 \%)$ & $\begin{array}{l}\text { Black et al., 2013; Boots et al., 2015; Cascioli et al., 2008; Diehl-Schmid } \\
\text { et al., 2013; Gibson et al., 2014; Griffiths and Bunyarong, 2016; Hughes } \\
\text { et al., 2014; Kerpershoek et al., 2017; Landmark et al., 2013; Lilly } \\
\text { et al., 2012; Nichols et al., 2009; Nicolaou et al., 2010; Peeters et al., } \\
\text { 2010; Peterson et al., 2016; Samia et al., 2012; Vaingankar et al., 2013; } \\
\text { Wawrziczny et al., 2017; Zwaanswijk et al., } 2013\end{array}$ \\
\hline \multicolumn{3}{|l|}{ 7. Relationships } \\
\hline a. With care recipient & $4(13 \%)$ & $\begin{array}{l}\text { Cascioli et al., 2008; Czarnuch and Mihailidis, 2011; Millenaar et al., } \\
\text { 2018; Wawrziczny et al., } 2017\end{array}$ \\
\hline b. With family & $7(23 \%)$ & $\begin{array}{l}\text { Gratão et al., 2010; Gibson et al., 2014; Griffiths and Bunyarong, 2016; } \\
\text { Landmark et al., 2013; Millenaar et al., 2018; Samia et al., 2012; } \\
\text { Wawrziczny et al., } 2017\end{array}$ \\
\hline \multicolumn{3}{|r|}{ (2) } \\
\hline a. Crises planning & $4(13 \%)$ & $\begin{array}{l}\text { Muangpaisan et al., 2010; Nicolaou et al., 2010; Vaingankar et al., 2013; } \\
\text { Wawrziczny et al., } 2017\end{array}$ \\
\hline b. Future planning & $7(23 \%)$ & $\begin{array}{l}\text { Cascioli et al., 2008; Gibson et al., 2014; Landmark et al., 2013; Lilly et al., } \\
\text { 2012; Millenaar et al., 2018; Samia et al., 2012; Wawrziczny et al., } 2017\end{array}$ \\
\hline $\begin{array}{l}\text { c. Information about } \\
\text { dementia and dementia } \\
\text { care }\end{array}$ & $16(52 \%)$ & $\begin{array}{l}\text { Black et al., 2013; Boots et al., 2015; Galvin et al., 2011; Gratão et al., } \\
\text { 2010; Gibson et al., 2014; Griffiths and Bunyarong, 2016; Hirakawa } \\
\text { et al., 2011; Jong and Boersma 2009; Kerpershoek et al., 2017; Lai and } \\
\text { Chung, 2007; Muangpaisan et al., 2010; Nicolaou et al., 2010; Peeters } \\
\text { et al., 2010; Peterson et al., 2016; Vaingankar et al., 2013; Zwaanswijk } \\
\text { et al., } 2013\end{array}$ \\
\hline $\begin{array}{l}\text { d. Information about } \\
\text { professional support and } \\
\text { formal services }\end{array}$ & $9(29 \%)$ & $\begin{array}{l}\text { Diehl-Schmid et al., 2013; Hirakawa et al., 2011; Lai and Chung, 2007; } \\
\text { Lilly et al., 2012; Muangpaisan et al., 2010; Peterson et al., 2016; Stirling } \\
\text { et al., 2010; Vaingankar et al., 2013; Zwaanswijk et al., } 2013\end{array}$ \\
\hline $\begin{array}{l}\text { e. Information about legal } \\
\text { regulation in caring }\end{array}$ & $3(10 \%)$ & Hirakawa et al., 2011; Vaingankar et al., 2013; Zwaanswijk et al., 2013 \\
\hline
\end{tabular}


Table 3. Suggested solutions put forward by primary authors to address caregivers' needs $(N=31)$

\begin{tabular}{|c|c|c|}
\hline SOLUTIONS FROM ARTICLES & $\begin{array}{l}\text { \# OF } \\
\text { STUDIES } \\
\quad(\%)\end{array}$ & REFERENCES \\
\hline \multicolumn{3}{|l|}{ Education and Information } \\
\hline $\begin{array}{l}\text { Educational activities about } \\
\text { dementia care according to } \\
\text { each stage of dementia } \\
\text { (practical advice) }\end{array}$ & $16(52 \%)$ & $\begin{array}{l}\text { Black et al., 2013; Cascioli et al., 2008; Czarnuch and Mihailidis, 2011; } \\
\text { Gratão et al., 2010; Hirakawa et al., 2011; Hughes et al., 2014; } \\
\text { Kerpershoek et al., 2017; Lawrence et al., 2008; McHugh et al., 2012; } \\
\text { Nichols et al., 2009; Nicolaou et al., 2010; Peterson et al., 2016; Rosa } \\
\text { et al., 2010; Samia et al., 2012; Vaingankar et al., 2013; Zwaanswijk } \\
\text { et al., 2013 }\end{array}$ \\
\hline $\begin{array}{l}\text { Information about coping with } \\
\text { behavioral problems and changes } \\
\text { in PWD }\end{array}$ & $15(48 \%)$ & $\begin{array}{l}\text { Boots et al., 2015; Cascioli et al., 2008; Gratão et al., 2010; Hirakawa } \\
\text { et al., 2011; Jong and Boersma, 2009; Kerpershoek et al., 2017; } \\
\text { Landmark et al., 2013; Lilly et al., 2012; McHugh et al., 2012; Nichols } \\
\text { et al., 2009; Nicolaou et al., 2010; Peeters et al., 2010; Raivio et al., } \\
\text { 2007; Rosa et al., 2010; Vaingankar et al., } 2013\end{array}$ \\
\hline $\begin{array}{l}\text { Information about dementia and } \\
\text { dementia care }\end{array}$ & $17(55 \%)$ & $\begin{array}{l}\text { Boots et al., 2015; Cascioli et al., 2008; Czarnuch and Mihailidis, 2011; } \\
\text { Diehl-Schmid et al., 2013; Galvin et al., 2011; Hirakawa et al., 2011; } \\
\text { Jong and Boersma, 2009; Kerpershoek et al., 2017; Lai and Chung, } \\
\text { 2007; Landmark et al., 2013; Nichols et al., 2009; Peeters et al., 2010; } \\
\text { Peterson et al., 2016; Raivio et al., 2007; Rosa et al., 2010; Vaingankar } \\
\text { et al., 2013; Wawrziczny et al., } 2017\end{array}$ \\
\hline Information about formal support & $8(26 \%)$ & $\begin{array}{l}\text { Black et al., 2013; Galvin et al., 2011; Hirakawa et al., 2011; Lai and } \\
\text { Chung, 2007; Nicolaou et al., 2010; Peterson et al., 2016; Raivio } \\
\text { et al., 2007; Stirling et al., } 2010\end{array}$ \\
\hline $\begin{array}{l}\text { Information provided in one central } \\
\text { place }\end{array}$ & $3(10 \%)$ & $\begin{array}{l}\text { Cascioli et al., 2008; Diehl-Schmid et al., 2013; Griffiths and Bunyarong, } \\
\quad 2016\end{array}$ \\
\hline \multicolumn{3}{|l|}{ Services } \\
\hline $\begin{array}{l}\text { Provide formal support related to } \\
\text { PWD health to informal caregivers } \\
\text { (e.g., home-care, services available } \\
\text { close to home, etc.) }\end{array}$ & $16(52 \%)$ & $\begin{array}{l}\text { Cascioli et al., 2008; Diehl-Schmid et al., 2013; Galvin et al., 2011; Gratão } \\
\text { et al., 2010; Griffiths and Bunyarong, 2016; Hughes et al., 2014; } \\
\text { Landmark et al., 2013; Lawrence et al., 2008; Lilly et al., 2012; } \\
\text { McHugh et al., 2012; Millenaar et al., 2018; Raivio et al., 2007; Rosa } \\
\text { et al., 2010; Vaingankar et al., 2013; Zwaanswijk et al., 2013; } \\
\text { Wawrziczny et al., } 2017\end{array}$ \\
\hline $\begin{array}{l}\text { Provide services to improve } \\
\text { caregivers' mental health } \\
\text { (emotional support) }\end{array}$ & $13(42 \%)$ & $\begin{array}{l}\text { Black et al., 2013; Boots et al., 2015; Cascioli et al., 2008; Galvin et al., } \\
\text { 2011; Hirakawa et al., 2011; Hughes et al., 2014; Lawrence et al., 2008; } \\
\text { Lilly et al., 2012; Muangpaisan et al., 2010; Nichols et al., 2009; Raivio } \\
\text { et al., 2007; Vaingankar et al., 2013; Wawrziczny et al., } 2017 .\end{array}$ \\
\hline $\begin{array}{l}\text { Provide daycare centers for PWD } \\
\text { that give personal time for } \\
\text { caregivers }\end{array}$ & $7(23 \%)$ & $\begin{array}{l}\text { Boots et al., 2015; Cascioli et al., 2008; Galvin et al., 2011; McHugh } \\
\text { et al., 2012; Millenaar et al., 2018; Wawrziczny et al., 2017; Vaingankar } \\
\text { et al., } 2013\end{array}$ \\
\hline $\begin{array}{l}\text { Differentiate the type of dementia } \\
\text { when supporting caregivers' } \\
\text { wellbeing }\end{array}$ & $2(6 \%)$ & Gibson et al., 2014; Nicolaou et al., 2010 \\
\hline
\end{tabular}

This review also highlighted two important gaps in the reporting of caregiver needs. Less than half of the studies reviewed reported on caregivers living arrangements, and three-quarters did not report on the type of dementia of the care recipient. These reporting gaps are troubling. A substantive body of research has established that co-residing and being a spousal caregiver are significant predictors of caregiver distress (e.g., Brodaty and Donkin, 2009). Recent findings have also underscored the unique implications of different types of dementia on caregiver tasks and subsequent level of burden (Cheng,
2017). Lack of reporting on these critical dimensions of the caregiving experience makes it difficult to compare, contrast, and contextualize caregivers' needs appropriately. As the academic literature around caregiver needs is quickly evolving, consensus around and consistent reporting on a common set of socio-demographic factors would advance comparative enquiry. Scaling up (or down) of services from one caregiver population to another should be informed by context-specific analysis of which living arrangements and type of dementia play significant roles. Primary study authors have an important role 
in ensuring the literature is complete and maximizing opportunities for secondary analysis.

Finally, the four largest studies in our review, representing $62 \%$ of all caregivers captured in our review, were conducted in United States, Finland, and Netherlands. It is crucial that large-scale studies using validated instruments are undertaken in other countries to ensure perspectives on caregivers' needs that are driving service development reflect the cultural, political, and social realities of caregivers in their own contexts. Updating the knowledge base on these issues is vital as some of the foremost reviews on racial and cultural differences in caregivers' experiences (e.g., Connell and Gibson, 1997; Janevic and Connell, 2001) are now almost two decades old. With only one study in our review comparing needs across multiple countries, there appears an opportunity to advance the understandings of caregivers' needs across different contexts.

\section{What types of caregiver needs are being reported in the literature?}

Given previous findings that the mean number of unique needs reported by caregivers of people with dementia is 10 (Miranda-Castillo et al., 2013), it was not surprising that this review found significant heterogeneity within and across studies. Consistent with other reviews, the most frequently cited need was related to caregivers' personal health, in particular their emotional health. The results from this scoping review align with previous literature showing the limited self-care performed by caregivers once they begin caring for a loved one (Ferreira et al., 2017; Keefe et al., 2008; Pinto and Barham, 2014; Schulz and Martire 2004; Tomomitsu et al., 2014). The impact on personal health is directly related to lack of help from others (Pinto et al., 2016). When caregivers do not receive help from others, they do not have time for self-care, which can contribute to perceived burden, higher probability of depression, and worse quality of life (Pinto et al., 2016; Yikilkan et al., 2014).

The emphasis in primary studies on informational needs underscores the learning processes that are inherent in the role of caregiving and their evolution over time. Caregivers who don't know what to expect about the disease prognosis or where to access formal support can feel overburdened because they do not have the information necessary to better manage care or to plan for assistance they may need in the future (McCabe et al., 2016).

The needs reported least often were related to coordination and housing. Juggling responsibilities was not reported as an issue in any of the primary studies in this review. One possible explanation for this is that researchers solicited information from caregivers on the outcomes (i.e., feeling burdened or depressed), not information on the reasons why those outcomes were occurring (i.e., feeling depressed because they were juggling too many responsibilities). Accordingly, attention should therefore be given to measures that capture outcomes and factors contributing to them. It may be that housing is largely a stable experience for caregivers, except for specific events when their needs in this area peak (e.g., transition of care recipient from home to long-term care). Single-data point data collection may not be capturing these lowprevalence events. Thus, future work needs to capture both prevalence and incidence longitudinallyacross the caregiver journey. Cohort and time series designs should measure not only changes in caregivers' psychological distress and quality of life (e.g., Bleijlevens et al., 2014), but also changes in caregiver needs to provide insight into services needed along the caregiving trajectory. Given the limited descriptions of actual interview questions and survey items, it was not possible to explore how the way questions were posed might have contributed to these low frequencies. The application of the C.A.R.E. Tool domains to categorize needs provided a useful lens for a broad scope of primary studies. All C.A.R.E. Tool domains, except for "juggling responsibilities," had at least one primary study in which it was identified by caregivers.

\section{What solutions and services are proposed to meet the needs of caregivers?}

In the face of wide-ranging needs and complex caregiving contexts, it was not entirely unexpected that recommendations made by primary study authors would be fairly abstract and imprecise. Many studies recommended "more information" must be provided to caregivers, with some providing topical areas in which information might be useful: self-care, relationship management with others involved in caring, social interactions (communication, empathy, control of aggressiveness, and assertiveness), and general information (dementia prognosis, stages of dementia, behavior changes, and formal support available). Three studies recommended that information be located in one central place, suggesting that the accessibility of reliable information could be lacking. Other studies identifying caregivers' informational needs indicated a demand for information by type and stage of dementia. Taken together, these recommendations could signal a need for more targeted, tailored resources to support caregivers in finding information relevant to their needs. Importantly, results of this review suggest that while we have some broad areas of general consensus around caregivers' informational needs, 
considerable work is needed to move from the broad to the specific. Evidence suggests tailored psychoeducational interventions are particularly effective in reducing caregiver burden (Beinart et al., 2012). Mapping specific caregiver needs, such as those identified in this review, to specific services, however, remains challenging. Realist methodologies that have recently been applied to caregiver intervention research (e.g., Parkinson et al., 2017) may also be useful in improving our understanding of needs. Rather than focusing on "what are the needs?" realist methods would explore "what needs, for whom, in what circumstances, and in what respects?." The findings of this scoping review suggest further "unpacking" of caregiver needs via these methods is warranted.

Access to formal supports was itself a common recommendation. Authors postulated that services such as day care centers, home visits, and service navigators might be helpful, as well as merging the services offered to caregivers and care recipients into one place (e.g., Diehl-Schmid, et al., 2013). In the same vein, recommendations for expanding the capacity of formal services included the delivery of a service model that combines the assistance of health professionals and community to create sustainable, ongoing support for caregivers. Having access to a professional navigator of the care system for caregivers of persons with dementia, for instance, would greatly benefit the sustainability of the caregiving situation. For example, if the person with Frontotemporal Dementia (FTD) is physically resisting the caregiver when attempting to bathe, caregivers would benefit from access to health professionals for clinical understanding and approaches to support the individual with FTD. In addition, community-based educational support resources about specific types of dementia would be helpful. Nicolaou et al. (2010) calls for the need to develop specific supports and education for caregivers of individuals with FTD because of their unique needs in comparison to other types of dementia. Knowledge translation will prove invaluable as we continue to take what is known about caregiver needs and realize realworld practices and policies for this population.

\section{Limitations}

This review included studies from different countries, but all of them have been published in English (or in both Portuguese and English). Therefore, we may not have captured the needs of caregivers from countries outside of the United States and Europe that were not published in the selected languages. Descriptors and key terms relevant to this study are wide-ranging. We acknowledge that some relevant articles may not have been captured as a function of our key search term selection. However, this search was informed by an experienced librarian and guided by search terms used in other relevant reviews. We did not assess the methodological quality of included studies, and while this limits the reliability and generalizability of findings, our approach is consistent with most published scoping reviews. We limited our search to peer-reviewed articles and reported on key methodological characteristics to facilitate some level of critical appraisal.

\section{Conclusions}

Understanding the needs of caregivers is vital to developing and delivering the kinds of services and resources that will alleviate burden and improve quality of life. As this body of research matures, it will need to advance the validity of instruments used to collect data on caregiver needs, report consistently on dimensions of caregivers' experiences well-known to mediate levels of burden, and explore in earnest not just what the needs are but how, when, and where those needs manifest across the caregiver journey.

\section{Conflict of interest}

None.

\section{Description of authors' roles}

All authors contributed to the conception and design of the review and interpretation of the results. FQ and EK independently reviewed and selected studies for inclusion, conducted data extraction, and checking. FQ wrote the manuscript with assistance from EK, and all authors provided substantive edits. All authors read and approved the final manuscript.

\section{Acknowledgements}

This research was supported by Canadian Institutes of Health Research Foundation Grant Program (FS2 201509FDN-IWKI-20675) and by São Paulo Research Foundation (FAPESP Grant \#2017/ 13807-0). The authors gratefully acknowledge Lucy Knight for her editorial assistance.

\section{Supplementary material}

To view supplementary material for this article, please visit https://doi.org/10.1017/S1041610219000243 


\section{References}

Afram, B., Verbeek, H., Bleijlevens, M. H. C. and Hammers, J. P. H. (2015). Needs of informal caregivers during transition from home towards institutional care in dementia: A systematic review of qualitative studies. International Psychogeriatrics, 27, 891-902. doi: 10.1017/ S1041610214002154.

Alzheimer's Association (2012). Alzheimer's disease facts and figures. Alzheimer's $\mathcal{E}$ Dementia, 8, 131-68. doi: 10.1016/j.jalz.2012.02.001.

Arksey, H. and O'Malley, L. (2005). Scoping studies: Towards a methodological framework. International fournal of Social Research Methodology: Theory E Practice, 8, 19-32. doi: 10.1080/1364557032000119616.

Bangerter, L. R., Griffin, J. M., Zarit, S. H. and Havyer, R. (2017). Measuring the needs of family caregivers or people with dementia: An assessment of current methodological strategies and key recommendations. Fournal of Applied Gerontology, 1-15. doi: 10.1177/ 0733464817705959.

Bardin, L. (1977). L'Analyse de contenu. France: Presses Universitaires de France.

Beinart, N., Weinman, J., Wade, D. and Brady, R. (2012). Caregiver burden and psychoeducational interventions in Alzheimer's disease: A review. Dementia and Geriatric Cognitive Disorders, 2, 638-648. doi: 10.1159/ 000345777.

Black, B. S., Johnston, D., Handel, S., Morrison, A., Robbins, B. and Rye, R. (2008). Manual for the fohns Hopkins Dementia Care Needs Assessment ( $F H D C N A$ ). Baltimore, MD: Self-published.

Black, B. S., Johnston, D., Rabins, P. V, Morrison, A., Lyketsos, C. and Samus, Q. M. (2013). Unmet needs of community-residing persons with dementia and their informal caregivers: Findings from the maximizing independence at home study. Fournal of the American Geriatrics Society, 61, 2087-2095. doi: 10.1111/jgs. 12549.

Bleijlevens, M. H. C., et al. (2014). Changes in caregiver burden and health-related quality of life of informal caregivers of older people with dementia: Evidence from the European Right Time Place CARE prospective cohort study. Fournal of Advanced Nursing, 71, 1378-1391.

Boots, L. M., Wolfs, C. A., Verhey, F. R., Kempen, G. I. and de Vugt, M. E. (2015). Qualitative study on needs and wishes of early-stage dementia caregivers: The paradox between needing and accepting help. International Psychogeriatrics, 27, 927-936. doi: 10.1017/ S1041610214002804.

Brodaty, H. and Donkin, M. (2009). Family caregivers of people with dementia. Dialogues in Clinical Neuroscience, 11, 217-228.

Cascioli, T. R., Al-Madfai, H., Oborne, P. and Phelps, S. (2008). An evaluation of the needs and service usage of family carers of people with dementia. Quality in Ageing, 9, 18-27. doi: 10.1108/14717794200800011.

Cheng, S. T. (2017). Dementia caregiver burden: A research update and critical analysis. Current Psychiatry Reports, 19, 64. doi: 10.1007/s11920-017-0818-2.

Connell, C. M., and Gibson, G. D. (1997). Racial, ethnic, and cultural differences in dementia caregiving: review and analysis. The Gerontologist, 37, 355-364.
Cooper, C., Balamuri, T. and Livingston, G. (2007). A systematic review of the prevalence and covariates of anxiety in caregivers of people with dementia. International Psychogeriatrics, 19, 175-195. doi: 10.1017/ S1041610206004297.

Crellin, N. E., Orrell, M., McDermott, O. and Charlesworth, G. (2014). Self-efficacy and health-related quality of life in family carers of people with dementia: A systematic review. Aging $\mathcal{E}$ Mental Health, 18, 954-969. doi: 10.1080/13607863.2014.915921.

Czarnuch, S. and Mihailidis, A. (2011). The design of intelligent in-home assistive technologies: Assessing the needs of older adults with dementia and their caregivers. Gerontechnology, 10, 169-182. doi: 10.4017/gt.2011.10.3 .005 .00 .

Diehl-Schmid, J., et al. (2013). Caregiver burden and needs in frontotemporal dementia. Fournal of Geriatric Psychiatry \& Neurology, 26, 221-229. doi: 10.1177/ 0891988713498467.

Elvish, R., Lever, S., Johnstone, J., Cawley, R. and Keady, J. (2013). Psychological interventions for carers of people with dementia: A systematic review of quantitative and qualitative evidence. Counselling and Psychotherapy Research, 13, 106-125. doi: 10.1080/14733145 .2012.739632.

Farina, N., et $\boldsymbol{a l}$. (2017). Factors associated with the quality of life of family carers of people with dementia: A systematic review. Alzheimer's Eீ Dementia, 13, 572-581. doi: 10 $.1016 / j$.jalz.2016.12.010.

Ferreira, C. R., Queluz, F. N. F. R., Ximenes, V. S., Isaac, L. and Barham, E. (2017). P3Es e a diminuição da sobrecarga em cuidadores: Confirmando efeitos em curto e longo prazo [Reducing burden among caregivers using the 3CP: Confirming short and long-term effects]. Revista Kairós-Gerontologia, 20, 131-150. doi: 10.23925/2176901X.2017v20i3p131-150.

Galvin, J. E., Duda, J. E., Kaufer, D. I., Lippa, C. F., Taylor, A. and Zarit, S. H. (2011). Lewy body dementia: Caregiver burden and unmet needs. Alzheimer Disease and Associated Disorders, 24, 177-181. doi: 10.1097/WAD $.0 \mathrm{~b} 013 \mathrm{e} 3181 \mathrm{c} 72 \mathrm{~b} 5 \mathrm{~d}$.

Gibson, A. K., Anderson, K. A. and Acocks, S. (2014). Exploring the service and support needs of families with early-onset Alzheimer's disease. American fournal of Alzheimer's Disease, 29, 596-600. doi: 10.1177/ 1533317514558160.

Gratão, A. C. M., et al. (2010). The demands of family caregivers of elderly individuals with dementia. Revista da Escola de Enfermagem da USP, 44, 873-880. doi: 10.1590/ S0080-62342010000400003.

Griffiths, J. and Bunrayong, W. (2016). Problems and needs in helping older people with dementia with daily activities: Perspectives of Thai caregivers. British fournal of Occupational Therapy, 79, 78-84. doi: 10.1177/ 0308022615604646.

Guberman, N., Keefe, J. and Fancey, P. (2018). The assessment experience of spousal dementia care-givers: 'It's made me realize that I am a person also'. Ageing $\mathcal{E}$ Society. Available at:https://www.cambridge.org/core/ journals/ageing-and-society/article/assessment-experienceof-spousal-dementia-caregivers-its-made-me-realise-that-iam-a-person-also; last accessed on 15 December 2018. 
Hirakawa, Y., Kuzuya, M., Enoki, H. and Uemura, K. (2011). Information needs and sources of family caregivers of home elderly patients. Archives of Gerontology $\mathcal{E}$ Geriatrics, 52, 202-205. doi: 10.1016/j .archger.2010.03.019.

Hughes, T. B., Black, B. S., Albert, M., Gitlin, L. N., Johnson, D. M., Lyketsos, C. G. and Samus, Q. M. (2014). Correlates of objective and subjective measures of caregiver burden among dementia caregivers: Influence of unmet patient and caregiver dementia-related care needs. International Psychogeriatrics, 26, 1875-1883. doi: 10.1017/ S1041610214001240.

Janevic, M. R. and Connell, C. M. (2001). Racial, ethnic, and cultural differences in the dementia caregiving experience: Recent findings. The Gerontologist, 41, 334-347.

Jong, J. D. and Boersma, F. (2009). Dutch psychogeriatric day-care centers: A qualitative study of the needs and wishes of carers. International Psychogeriatrics, 21, 268-277. doi: $10.1017 /$ S1041610208008247.

Keefe, J., Guberman, N., Fancey, P., Barylak, L. and Nahmiash, D. (2008). Caregivers' Aspirations, Realities, and Expectations: The C.A.R.E. Tool. Fournal of Applied Gerontology, 27, 286-308. doi: 10.1177/ 0733464807312236.

Kerpershoek, L., et al. (2017). Needs and quality of life of people with middle-stage dementia and their family carers from the European Actifcare study. When informal care alone may not suffice. Aging $\mathcal{E}$ Mental Health, 1, 1-6. doi: 10 $.1080 / 13607863.2017 .1390732$.

Lai, C. K. Y. and Chung, J. C. C. (2007). Caregivers' informational needs on dementia and dementia care. Asian Fournal of Gerontology \& Geriatrics, 2, 78-87.

Laks, J., Goren, A., Dueñas, H., Novick, D. and Kahle-Wrobleski, K. (2016). Caregiving for patients with Alzheimer's disease or dementia and its association with psychiatric and clinical comorbidities and other health outcomes in Brazil. International fournal of Geriatric Psychiatry, 31, 176-185. doi: 10.1002/gps.4309.

Landmark, B. T., Aasgaard, H. S. and Fagerström, L. (2013). "To be stuck in it-I can't just leave": A qualitative study of relatives' experiences of dementia suffers living at home and need for support. Home Health Care Management $\mathcal{E}$ Practice, 25, 217-223. doi: 10.1177/ 1084822313487984.

Lawrence, V., Murray, J., Samsi, K. and Banerjee, S. (2008). Attitudes and support needs of Black Caribbean, south Asian and White British carers of people with dementia in the UK. The British fournal of Psychiatry, 193, 240-246. doi: 10.1192/bjp.bp.107.045187.

Lilly, M. B., Robinson, C. A., Holtzman, S. and Bottorff, J. L. (2012). Can we move beyond burden and burnout to support the health and wellness of family caregivers to persons with dementia? Evidence from British Columbia, Canada. Health and Social Care in the Community, 20, 103-112. doi: 10.1111/j.1365-2524.2011 $.01025 . x$.

Mansfield, E., Boyes, A. W. and Sanson-Fisher, R. (2017). Quantifying the unmet needs of caregivers of people with dementia: A critical review of the quality of measures. International fournal of Geriatric Psychiatry, 32, 274-287. doi: $10.1002 /$ gps.4642.
McCabe, M., You, E. and Tatangelo, G. (2016). Hearing their voice: A systematic review of dementia family caregivers' needs. The Gerontologist, 56, 70-88. doi: 10 .1093/geront/gnw078.

McHugh, J., Wherton, J., Prendergast, D. and Lawlor, B. (2012). Identifying opportunities for supporting caregivers of persons with dementia through information and communication technology. Gerontechnology, 10, 220-230. doi: 10.4017/gt.2012.10.4 .003 .00

Millenaar, J. K., et al. (2018). Exploring perspectives of young onset dementia caregivers with high versus low unmet needs. International fournal of Geriatric Psychiatry, 33, 340-347. doi: 10.1002/gps.4749.

Miranda-Castillo, C., Woods, B. and Orrell, M. (2013). The needs of people with dementia living at home from user, caregiver and professional perspectives: a cross-sectional survey. BMC Health Services Research, 13, 13-43. doi: 10.1186/1472-6963-13-43.

Moher, D., Liberati, A., Tetzlaff, J., Altman, D. G. and The Prisma Group. (2009). Preferred Reporting Items for Systematic Reviews and Meta-Analyses: The PRISMA Statement. PLOS Medicine, 6, e1000097. doi: 10.1371/ journal.pmed.1000097.

Muangpaisan, W., Assanasen, J., Assantachai, P., Chatthanawaree, W. and Kuptnirasaikul, V. (2010). Caregiver burden and needs of dementia caregivers in Thailand: a cross-sectional study. Fournal of the Medical Association of Thailand, 93, 601-607.

Nichols, L. O., Martindale-Adams, J., Greene, W.A., Burns, R., Graney, M. J. and Lummus, A. (2009). Dementia caregivers' most pressing concerns. Clinical Gerontologist, 32, 1-14. doi: 10.1080/0731711080 2468546.

Nicolaou, P. L., Egan, S. J., Gasson, N. and Kane, R. T. (2010). Identifying needs, burden, and distress of carers of people with frontotemporal dementia compared to Alzheimer's disease. Dementia, 9, 215-235. doi: 10.1177/ 1471301209354024.

Oken, B. S., Fonareva, I. F. and Wahbeh, H. (2011). Stress-related cognitive dysfunction in dementia caregivers. fournal of Geriatric Psychiatry, 24, 191-198. doi: 10.1177/ 0891988711422524.

Orrell, M. and Hancock, G. (2004) CANE: Camberwell Assessment of Need for the Elderly. London, UK: Gaskell.

Parkinson, M., Carr, S. M., Rushmer, R. and Abley, C. (2017). Investigating what works to support family carers of people with dementia: A rapid realist review. Fournal of Public Health, 39, e290-e301. doi: 10.1093/ pubmed/fdw100.

Peeters, J. M., Van Beek, A. P. A., Meerveld, J. H. C. M., Spreeuwenberg, P. M. M. and Francke, A. L. (2010). Informal caregivers of persons with dementia, their use of and needs for specific professional support: A survey of the national dementia programme. BMC Nursing, 9, 9. doi: 10.1186/1472-6955-9-9.

Peterson, K., Hahn, H., Lee, A. J., Madison, C. A. and Atri, A. (2016). In the informational age, do dementia caregivers get the information they need? Semi-structured interviews to determine informal caregivers' education needs, barriers, and preferences. BMC Geriatrics, 16, 1-13. doi: 10.1186/s12877-016-0338-7. 
Pinto, F. N. F. R. and Barham, E. J. (2014). Bem-estar psicológico: Comparação entre cuidadores de idosos com e sem demência [Psychological wellbeing: A comparison between caregivers of older adults, with and without dementia]. Psicologia, Saúde E Doenças, 15, 635-655. doi: 10.15309/14psd150307.

Pinto, F. N. F. R., Barham, E. J. and Del Prette, Z. A. P. (2016). Interpersonal conflicts among family caregivers of the elderly: The importance of social skills. Paidéia (Ribeirão Preto), 26, 161-170. doi: 10.1590/198243272664201605.

Posner, B., Sutter, M., Perrin, P. B., Ramirez Hoyos, G., Arabia Buraye, J. and Arango-Lasprilla, J. C. (2015). Comparing dementia caregivers and healthy controls in mental health and health related quality of life in Cali, Colombia. Psicología desde el Caribe, 32, 1-26. doi: 10.14482/psdc.32.1.6273.

Quinn, C., Clare, L. and Woods, R. T. (2010). The impact of motivations and meanings on the well-being of caregivers of people with dementia: A systematic review. International Psychogeriatrics, 22, 43-55. doi: 10.1017/ S1041610209990810.

Raivio, M., et al. (2007). How do officially organized services meet the needs of elderly caregivers and their spouses with Alzheimer's disease? American fournal of Alzheimer's Disease and Other Dementias, 22, 360-368. doi: 10.1177/ 1533317507305178.

Rosa, E., Lussignoli, G., Sabbatini, F., Chiappa, A., S, D. C., Lamanna, L., and Zanetti, O. (2010). Needs of caregivers of the patients with dementia. Archives of Gerontology $\mathcal{E}$ Geriatrics, 51, 54-58. doi: 10.1016/j.archger .2009.07.008.

Samia, L. W., Hepburn, K. and Nichols, L. (2012). "Flying by the seat of our pants": What dementia family caregivers want in an advanced caregiver training program. Research in Nursing and Health, 35, 598-609. doi: 10.1002/ nur. 21504.

Schulz, R. and Martire, L. M. (2004). Family caregiving of persons with dementia: prevalence, health effects, and support strategies. The American fournal of Geriatric Psychiatry, 12, 240-249.

Sörensen, S. and Conwell, Y. (2011). Issues in dementia caregiving: Effects on mental and physical health, intervention strategies, and research needs. American Fournal of Geriatric Psychiatry, 19, 491-496. doi: 10.1097/ JGP.0b013e31821c0e6e.

Stirling, C., Andrews, S., Croft, T., Vickers, J., Turner, P. and Robinson, A. (2010). Measuring dementia carers' unmet need for services - An exploratory mixed method study. BMC Health Services Research, 10, 122. doi: 10.1186/ 1472-6963-10-122.

Takai, M., Takahashi, M., Iwamitsu, Y., Oishi, S. and Miyaoka, H. (2011). Subjective experiences of family caregivers of patients with dementia as predictive factors of quality of life. Psychogeriatrics, 11, 98-104. doi: 10.1111/j .1479-8301.2011.00354.x.

Tomomitsu, M. R. S. V., Perracini, M. R. and Neri, A. L. (2014). Fatores associados à satisfação com a vida em idosos cuidadores e não cuidadores [Factors associated with life satisfaction among elderly people who are caregivers and non-caregivers]. Ciência E Saúde Coletiva, 19, 3429-3440. doi: 10.1590/1413-81232014198.13952013.

Vaingankar, J. A., et al. (2013). Perceived unmet needs of informal caregivers of people with dementia in Singapore. International Psychogeriatrics, 25, 1605-1619. doi: 10.1017/ S1041610213001051.

Waligora, K., Bahouth, M. and Han, H. (2018). The selfcare needs and behaviors of dementia informal caregivers: A systematic review. The Gerontologist, 1-9. doi: 10.1093/ geront/gny076.

Wawrziczny, E., Pasquier, F., Ducharme, F., Kergoat, M. J. and Antoine, P. (2017). Do spouse caregivers of young and older persons with dementia have different needs? A comparative study. Psychogeriatrics, 17, 282-291. doi: 10.1111 psyg. 12234 .

Whitlatch, C. J. and Orsulic-Jeras, S. (2018). Meeting the informational, educational, and psychosocial support needs of persons living with dementia and their family caregivers. The Gerontologist, 58, S58-S73. doi: 10.1093/geront/gnx162.

World Health Organization (WHO) (2017). Global action plan on the public health response to dementia 2017-2025. Geneva: WHO Document Production Services.

World Health Organization (WHO) (2018). Dementia: mhGAP training of healthcare providers training manual and supporting material. Geneva: WHO Document Production Services.

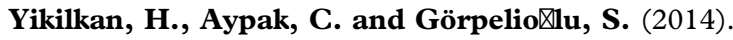
Depression, anxiety and quality of life in caregivers of longterm home care patients. Archives of Psychiatric Nursing, 28, 193-196. doi: 10.1016/j.apnu.2014.01.001.

Zwaanswijk, M., Peeters, J. M., van Beek, A. P., Meerveld, J. H. and Francke, A. L. (2013). Informal caregivers of people with dementia: problems, needs and support in the initial stage and in subsequent stages of dementia: A questionnaire survey. Open Nursing fournal, 7, 6-13. doi: 10.2174/1874434601307010006. 